\title{
General Psychiatry Development of a psychosocial functioning questionnaire for patients with major depressive disorder
}

\author{
Xiujuan Zhang, Hua Yang, Hui Ma, Changjun Teng, Hui Wang, Kaili Diao, \\ Ning Zhang
}

To cite: Zhang $X$, Yang $\mathrm{H}$, Ma $\mathrm{H}$, et al. Development of a psychosocial functioning questionnaire for patients with major depressive disorder. General Psychiatry 2022;35:e100527. doi:10.1136/ gpsych-2021-100527

$\mathrm{XZ}$ and $\mathrm{HY}$ are joint first authors.

Received 30 March 2021 Accepted 27 January 2022

Check for updates

(c) Author(s) (or their employer(s)) 2022. Re-use permitted under CC BY-NC. No commercial re-use. See rights and permissions. Published by BMJ.

Department of Medical Psychology, Nanjing Brain Hospital Affiliated to Nanjing Medical University, Nanjing, Jiangsu, China

Correspondence to

Dr Ning Zhang;

zn6360@126.com

\section{ABSTRACT}

Background The importance of psychosocial functioning in the assessment, diagnosis and treatment of major depressive disorder (MDD) is widely recognised. However, there is a lack of effective scales to assess psychosocial functioning in patients with MDD.

Aims To develop a professional questionnaire to evaluate the psychosocial functioning of patients with MDD.

Methods Using a literature review, an open-ended questionnaire survey, and patient interviews, a theoretical model of psychosocial functioning was constructed, and an initial questionnaire was formed which included four dimensions. After two rounds of testing, using items analysis and exploratory factor analysis, a finalized questionnaire was created. There were 460 patients with MDD selected from six psychiatric hospitals for formal testing using a convenience sampling method. Forty patients were randomly selected and retested one week later to evaluate the test-retest reliability of the scale. The Patient Health Questionnaire-9 (PHQ-9), Short Form of Quality of Life Enjoyment and Satisfaction Questionnaire (Q-LES-Q-SF), Sheehan Disability Scale (SDS), and Dysfunctional Attitudes Scale (DAS) provided major standards to check the criterion validity of this questionnaire. Correlation analysis, confirmatory factor analysis, and internal consistency reliability testing were used to examine other psychometric characteristics of the finalized questionnaire.

Results The Psychosocial Functioning Questionnaire (PFQ) for patients with MDD included three dimensions: psychological cognitive functioning, subjective wellbeing, and social functioning, with a total of 18 items. The overall internal consistency reliability of the questionnaire was 0.957 , and the test-retest reliability was 0.840 . Confirmatory factor analysis showed that the model fitted well: Goodness of Fit Index (GFI) $=0.888$; Root Mean SquareError of Approximation (RMSEA) $=0.085$. The total score of $P F Q$ was significantly correlated with the total score of PHQ-9, DAS, SDS and Q-LES-Q-SF ( $|r|=0.599$ $0.870, p<0.001$ ).

Conclusions The Psychosocial Functioning Questionnaire has good reliability and validity. It can be used to measure the psychosocial functioning of patients with MDD.

\section{INTROUDCTION}

The importance of psychosocial functioning in the assessment, diagnosis and treatment of

\section{Key messages}

What is already known on this topic

- Patients with major depressive disorder (MDD) have substantial impairment in psychosocial functioning, and the improvement of psychosocial functioning is strongly associated with relapse of MDD. But existing evaluation tools for psychosocial functioning do not precisely measure functional impairments that are unique to these groups, such as patients with MDD.

\section{What this study adds}

- This study concentrates on MDD patients' features of psychosocial functioning impairment and develops a psychosocial functioning questionnaire with good reliability and validity for patients with MDD.

\section{How this study might affect research, practice} or policy

- The psychosocial functioning questionnaire developed in this study helps to comprehensively assess the treatment, recovery, and relapse prevention of depression, thus advancing the quality of treatment and reducing the relapse rate.

major depressive disorder (MDD) is widely recognised. It has been found that patients with MDD have significant impairments in psychosocial functioning, and the impairments do not often entirely disappear even after patients achieve remission of depressive symptoms; ${ }^{12}$ this is closely related to the recurrence of MDD. ${ }^{3}$

The concept of psychosocial functioning can be traced back to 1963 when Katz et al defined psychosocial functioning as the activity of daily living. ${ }^{4}$ Yerxa et al (1967) highlighted individuals' social functioning: What do we want to do? Are we capable of doing it? What restricts the individual from doing something? ${ }^{5}$ In 1983, Feragne combined psychological functioning with psychosocial functioning, classified as subjective well-being and role functioning. ${ }^{6}$ Subsequently, different psychosocial functioning descriptions have 
emerged, but their common elements comprise both psychological functioning and social functioning. Many scholars have now modified their definitions of psychosocial functioning according to the theory introduced by Bonder in 1993. Bonder separated psychosocial functioning into psychological and social variables, with the social variables including skill components and professional performance. ${ }^{7}$

Some researchers have defined the psychosocial functioning of patients with MDD. In their research on postpartum depression, Whiffen et al classified patients' psychosocial functioning into three main dimensions: interpersonal relationship, stress, and coping styles. ${ }^{8}$ Since then, Clark et al in 2003 asserted that cognitive, social, and interpersonal functioning should be involved when assessing psychosocial functioning of depressed patients. ${ }^{9}$ Lam et al (2011) proposed that the assessment of psychosocial functioning of depressed patients must comprise the quality of life and social and occupational functioning; they determined psychosocial functioning to be an individual's ability to achieve life tasks and interact with others in a mutually satisfying manner. ${ }^{10}$

Thoughout the past several decades, studies of the psychosocial functioning of patients have used a variety of evaluation tools. The earliest questionnaire was the Psychosocial Functioning Inventory compiled by Ferragne in 1983. Later, in 1987, the American Psychiatric Association also developed the Global Assessment of Functioning (GAF). Other commonly used scales include the Sheehan Disability Scale (SDS), the 36-Item Short-Form Health Survey (SF-36), the Social and Occupational Functioning Assessment Scale (SOFAS), the WHO Quality of Life-100 (WHOQOL-100), the Social Functioning Questionnaire (SFQ), and the Social Adjustment Scale (SAS), and so on. These tools have good metrological properties but also include deficiencies. First, evaluation tools specific for diagnostic groups are lacking, and existing instruments do not precisely measure functional impairments that are unique to these groups, such as MDD patients. The connotation of psychosocial functioning depends upon the research focus. For example, when evaluating the psychosocial functioning of patients with severe mental illness, such as schizophrenia, social and basic functioning is considered, while when studying individuals without mental illness, the focus is on self-esteem, values, interests, and so on. ${ }^{11}$ Clark et al proposed that the evaluation of psychosocial functioning in patients with depression should include cognitive, social, and interpersonal functioning. ${ }^{9}$ Second, since no agreed-upon measure of psychosocial functioning in depressed patients exists, researchers have utilized various questionnaires and tools to evaluate this dimension. This disparity has limited the generalisability of the research results. Lastly, to varying degrees, many questionnaires confuse functioning and symptoms. For instance, GAF mainly measures the severity of symptoms rather than the level of psychosocial functioning. ${ }^{11}$

In summary, the need exists for a scale such as the Psychosocial Functioning Questionnaire (PFQ) for patients with depression that we have developed. Based on prior research findings, this article will concentrate on MDD patients' features of psychosocial functioning impairment and will propose a theoretical construction and standardised measurement of MDD patients' psychosocial functioning.

In conjunction with the development and evolution of the psychosocial functioning concept, this article defines psychosocial functioning in patients with MDD as the ability of an individual to create relationships with others and society in a mutually pleasing manner, and the ability to achieve a healthy life independently. Psychosocial functioning involves four dimensions: psychological cognitive functioning, subjective well-being, social functioning, and basic functioning. The first dimension is named psychocognitive functioning in order to distinguish it from neurocognitive functioning. Neurocognition refers to memory, attention, reaction time and so on, while psychological cognition refers to self-evaluation, self-control, attribution, expectation, and so on. To analyse and expand the connotation and structure of psychosocial functioning stated by the research literature, we developed an open-ended questionnaire and interviewed 12 representative patients with MDD (5 men and 7 women) in a psychiatric hospital. The questionnaires' content included: 'What do you think are functional impairments you experience because of depression?', and 'What is the essential psychosocial functioning that you anticipate recovering?' The outcomes revealed that the most common impairment noted was psychological cognitive functioning, such as 'worrying about the future', 'not being able to work normally' and 'feeling hopeless'. Combined with results from the openended questionnaire and one-on-one interviews, the four dimensions of the psychosocial functioning are further explained. Among them, psychological cognitive functioning includes depressed patients' self-evaluation, selfcontrol, beliefs and expectations, such as thinking that they are losers and the future is hopeless. Subjective well-being comprises the balance of positive and negative emotions and life satisfaction. Social functioning principally involves work performance, family relations, interpersonal relationships, personal life, and social participation. Finally, basic functioning primarily refers to physical activity, self-care, and health-imposed restrictions in physical functioning.

\section{METHODS}

\section{Subjects}

From January to December 2015, patients from psychiatric hospitals in Jiangsu Province, China, who met the MMD diagnostic criteria of the Diagnostic and Statistical Manual of Mental Disorders, Fifth Edition (DSM-5), were chosen by using convenience sampling. Considering the need for factor analysis, the sample size was greater than 100 participants (figure 1 ).

\section{Sample 1}

The first group was chosen for the administration and analysis of the initial questionnaire. Patients with MDD 


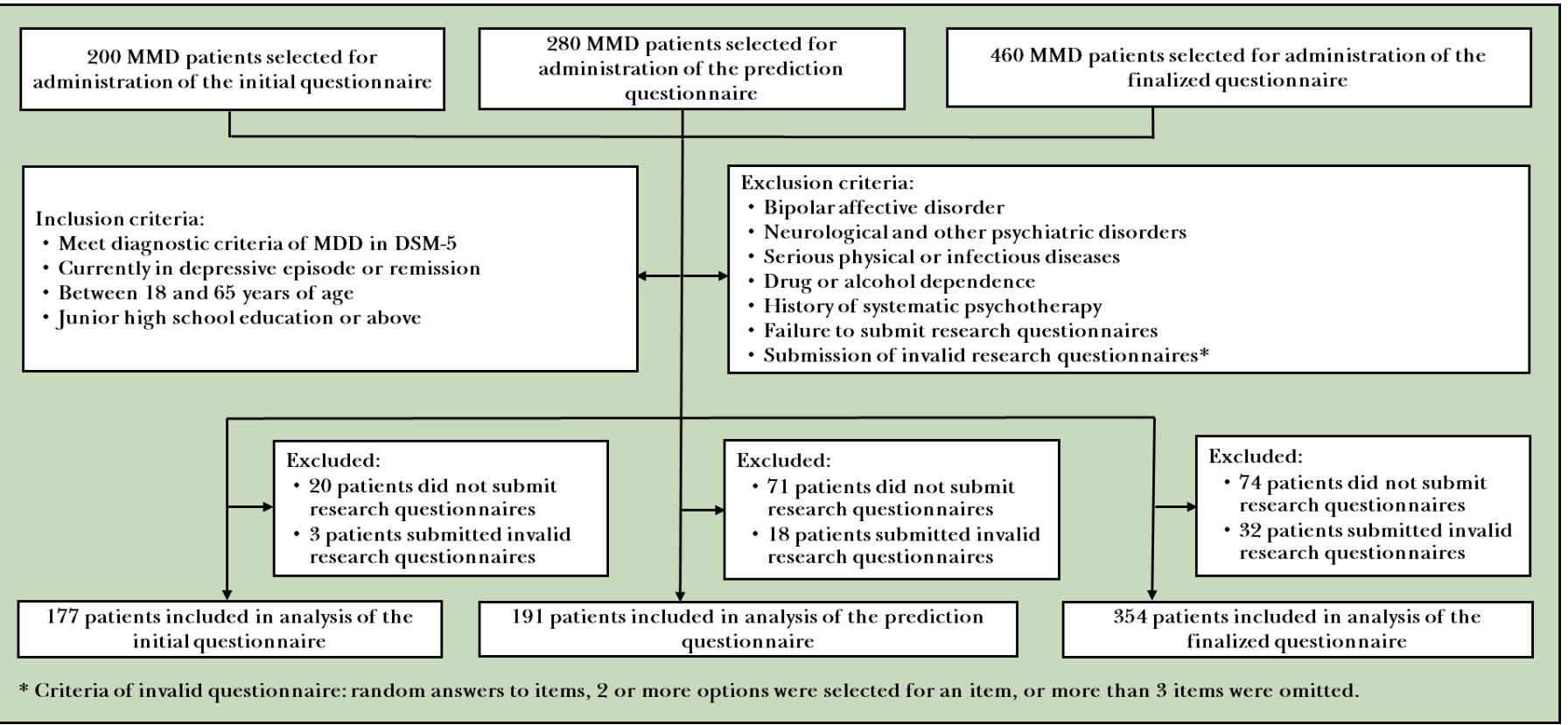

Figure 1 Flowchart for participant enrollment. DSM-5, Diagnostic and Statistical Manual of Mental Disorders, Fifth Edition; MMD, major depressive disorder.

from a psychiatric hospital were selected as study participants by using convenience sampling. A total of 200 questionnaires were distributed, 180 questionnaires were collected, and 177 of the latter were valid. The response rate was $90 \%$. There were 86 men and 91 women. The mean (SD) age was 42 (10) years old.

\section{Sample 2}

The second group was chosen for the administration and analysis of the prediction questionnaire. Patients with MDD from a psychiatric hospital were selected as study participants by using convenience sampling. A total of 280 questionnaires were distributed, 209 questionnaires were collected, and 191 of the latter were valid. The response rate was $75 \%$. There were 89 men and 102 women. The mean (SD) age was 38 (12) years old.

\section{Sample 3}

The third group was chosen for the administration and analysis of the finalized questionnaire. Patients with MDD from six psychiatric hospitals were selected as study participants by using convenience sampling. A total of 460 questionnaires were distributed, 386 questionnaires were recovered, and 354 of the latter were valid. The response rate was $84 \%$. There were 128 men and 226 women. The mean (SD) age was 41 (13) years old.

\section{Tools}

\section{Psychosocial Functioning Questionnaire}

Formation of the initial questionnaire

According to the four functional dimensions, including psychological cognitive functioning, subjective well-being, social functioning, and basic functioning, a pool of entries of the PFQ for patients with MDD was built by referring to questionnaires associated with functional measures that were more frequently adopted or more mature, such as GAF, SOFAS, SAS, SFQ, SF-36, WHOQOL-100, the Scale of Psychological Well-Being, the Satisfaction With Life Scale, and the Quality of Life Enjoyment and Satisfaction Questionnaire.

There were 92 self-assessment items in the pool, including 20, 25, 32, and 15 items in psychological cognitive functioning, subjective well-being, social functioning, and basic functioning dimensions, respectively. The scope of the social functioning dimension includes different fields such as family, work, and social networking, so the number of proposed items was the largest. The impairment of basic functioning in patients with MDD was relatively mild, so the number of proposed items was the least.

The questionnaire was designed as a self-assessment scale, using the 5-point Likert scale. The questionnaire had two scoring methods: forward scoring and reverse scoring, with one-third of the items using the latter method.

\section{Administration and analysis of the initial questionnaire}

The initial questionnaire was administered to sample 1 . To capture the participants' feedback on each item, oneon-one evaluation was adopted in this round of testing.

Initially, through exploratory factor analysis, the research team removed 47 items with a factor load of less than 0.3. The number of remaining items in the dimensions of psychological cognitive functioning, subjective well-being, social functioning, and basic functioning were 10, 13, 17 and 5, respectively. Next, the data analysis outcome and the retained items were reviewed by two professors specialising in medical psychology, three chief psychiatrists, one postdoctoral fellow in medical psychology, and one editor of a journal specialising in psychiatry. Because depression does 
not lead to significant impairment in basic functioning, such as being able to dress oneself or walking without falling. Elderly patients may have these difficulties, but these impairments are not common in the majority of individuals with depression. Therefore, finally, the research team removed all of the five items dealing with basic functioning.

After that, we analysed the remaining 40 items one by one. The entry criteria of items were as follows: the correlation coefficient between the items did not surpass 0.9 (for example, the content estimated by two items does not overlap); the correlation coefficients between the dimensions, between each item and the total questionnaire, between the dimension and items that the dimension includes, ranged from 0.3 to 0.8 . The effect of removing an entry on the overall structure of the questionnaire was continuously attempted. Four items in psychological cognitive functioning, four items in subjective well-being, and six items in social functioning were further removed. Consequently, the number of items in the prediction questionnaire composed after this round of administration was 26, including 6,9 , and 11 items in psychological cognitive functioning, subjective wellbeing, and social functioning, respectively.

\section{Test and analysis of the prediction questionnaire}

The prediction questionnaire was administered to sample 2 , and the data were analysed by items analysis and exploratory factor analysis. Thereby, a total of 8 inappropriate items were removed and 18 items were retained.

Among them, one item with a correlation coefficient of less than 0.3 with the questionnaire's total score was removed. Two items with insignificant difference between the high group (top 27\% of the total score) and the low group (bottom $27 \%$ of the total score) were removed in the test of significance of each item's mean difference. Three items with factor loadings less than 0.4 or similar loadings on numerous factors at the same time were deleted. The research used principal component analysis and the maximum variance rotation method to extract common factors. Considering that the interpretation rate of the extracted common factors to the variance of the original variable was greater than $60 \%$, four common factors were extracted in this study, explaining $65.51 \%$ of the total variation. The first three public factors corresponded to the social functioning, subjective well-being, and psychological cognitive dimension of the theoretical constructs, respectively. The two items of factor 4, 'I feel depressed, worried or uncomfortable at work or doing other things' and 'I feel optimistic and confident enough in the last 2 weeks' leaned more towards the symptom level. Considering that the questionnaire was used to measure psychosocial functioning, the fourth factor was eventually removed.

Above all, the theoretical concept, results of two rounds of measurement, experts' review opinions, and patients' feedback were combined to improve the items, structure, and language expression of the questionnaire, resulting in a finalized questionnaire consisting of 18 items in three dimensions: psychological cognitive functioning (including 4 items), subjective well-being (containing 5 items) and social functioning (comprising 9 items). The questionnaire was self-assessed in constructing a 5-point scale from 1 to 5 , with items 7, 10, 11, 12 and 13 being reverse scoring questions. The higher the questionnaire score, the more serious the impairment in psychosocial functioning and the lower the psychosocial function level.

\section{Validity test instruments}

Patient Health Questionnaire-9 (PHQ-9)

It's a 4-point self-assessment scale for depressed patients with 9 items. The total score ranges from 0 to 27 and the critical value is 4 . Higher scores indicate more severe depressive symptoms. The questionnaire has been shown to have high homogeneous reliability and test-retest reliability, ${ }^{12}$ with internal consistency reliability of 0.834 in this investigation.

\section{Short Form of Quality of Life Enjoyment and Satisfaction Questionnaire (Q-LES-Q-SF)}

This questionnaire comprises 16 self-assessment items with five grades of evaluation. Its dimensions involve physical health, subjective feelings, leisure activities, social relationships, general activities, satisfaction with medication and overall life satisfaction. The questionnaire has high-grade reliability and validity, ${ }^{13}$ with internal consistency reliability of 0.890 in this research.

\section{Sheehan Disability Scale}

It's a self-assessment scale consisting of three items: work/ school, social life, and family life. It uses a numerical scale between 0 and 10, with 0 representing no effect at all, $1-3$ being a mild effect, $4-6$ being a moderate effect, 7-9 being an obvious effect, and 10 being an extremely severe effect. The scale regularly takes the score of each item and the total score as indicators. It assesses disability and functional impairment caused by various chronic diseases, including mental disorders. Researchers have revealed that the scale has excellent reliability and validity, ${ }^{14}$ with internal consistency reliability of 0.918 in the present study.

\section{Dysfunctional Attitudes Scale (DAS)}

This is a 7-point self-assessment questionnaire largely employed to evaluate typical, persistent depressive attitudes or beliefs, with higher scores indicating higher cognitive impairment levels. There are 40 items in the questionnaire that can be summarised by eight factor structures: vulnerability, attraction/rejection, perfectionism, coercion, approval-seeking, dependence, autonomy attitude, and cognitive philosophy. The questionnaire has good reliability and validity in the Chinese population, ${ }^{15}$ with internal consistency reliability of 0.890 in this research.

\section{Self-compiled questions}

This is a self-compiled questionnaire with a single item,which uses a number from 1 to 7 to "show your 
current life functioning" ( $1=$ cannot live well, $7=$ can live well).

\section{Statistical methods}

SPSS 11.0 was used for correlation analysis, t-test and exploratory factor analysis of the data. AMOS V.21.0 software was used for confirmatory factor analysis.

Exploratory factor analysis and correlation analysis were carried out on the initial questionnaire and prediction questionnaire. Correlation analysis included calculating the correlation coefficients between item scores, between each item score and the total score, between each item score and the dimension score, and between dimension scores. T-test of two independent groups was used to examine the significance of the mean difference in the scores of each item between the high group (27\% before the total score) and the low group (27\% after the total score).

Cronbach correlation coefficient was adopted to analyse the internal consistency reliability of the finalized questionnaire. To test the finalized questionnaire's test-retest reliability, correlation analysis between the consequences of two administrations of the same population was used. The finalized questionnaire's construct validity was examined through confirmatory factor analysis, the correlation analysis between the questionnaire's dimensions and the total questionnaire. The finalized questionnaire's criterion validity was investigated by correlation analysis between the finalized questionnaire and the validity test instruments.

\section{RESULTS}

\section{Reliability test}

In this study, the Cronbach coefficient was used to examine the internal consistency reliability of the finalized questionnaire. The results shown in table 1 indicated a good internal consistency reliability, which ranged from 0.842 to 0.957 .

Forty patients with MDD were randomly selected from sample 3 for test-retest oneweek later. The Pearson correlation coefficient ranged from 0.698 to 0.840 , which proved that the test-retest reliability of the questionnaire was ideal.
Validity test

\section{Construct validity}

The structural equation model was established based on the measured data, and confirmatory factor analysis was carried out. There was no negative variance in the model, and the standardised coefficient was less than 0.95 , which indicated that there was no violation of estimation in the model, and the model evaluation process can be carried out. In the model correction, the modified index was obtained by releasing the relationship between the error terms and establishing the correlation between the error terms, and then the correction model was obtained. The fitting index of the data model was between 0.888 and 0.947 , and the root mean square error of approximation (RMSEA) value was 0.085 , which all reached the standard level (the fitting index was close to 1 , and the RMSEA value was less than 0.1), and proved that the structure of PFQ was effective. See table 2 for details.

The result of Pearson correlation analysis between dimensions of the questionnaire and between each dimension and the total questionnaire was shown in table 3. The data showed that the correlation coefficients between the three dimensions and the total questionnaire were all above 0.9 , and the correlation coefficients between dimensions were also above 0.8 .

\section{Criterion validity}

The Pearson correlation analysis between the selfcompiled questionnaire and the total PFQ showed that the correlation coefficient was $-0.765(p<0.001)$. In addition, the correlation coefficient between the total PFQ and the PHQ-9 was $0.789(\mathrm{p}<0.001)$.

According to the three dimensions of $\mathrm{PFQ}$, including subjective well-being, psychological cognitive functioning and social functioning, three classic questionnaires were selected to further explore the criterion validity of the new questionnaire: Q-LES-Q-SF, DAS, and SDS. The data distribution did not obey the normal distribution, so Spearman correlation analysis was used. Table 4 showed that the scores of the three questionnaires were significantly correlated with the scores of PFQ. Further comparison finded that the score of Q-LES-Q-SF had the highest correlation with the score of subjective well-being $(\mathrm{r}=-0.853)$, the score of DAS had the highest correlation with the score of psychological cognitive functioning $(\mathrm{r}=0.662)$, and

Table 1 Results of reliability tests

\begin{tabular}{|c|c|c|c|c|}
\hline Reliability & Subjective well-being & Psychological cognitive functioning & Social functioning & Total PFQ \\
\hline $\begin{array}{l}\text { Internal consistency } \\
\text { reliability }\end{array}$ & $0.945^{\star \star \star}$ & $0.842^{\star \star \star}$ & $0.899^{\star \star \star}$ & $0.957^{\star * *}$ \\
\hline
\end{tabular}

${ }^{* \star *} \mathrm{p}<0.001$.

PFQ, Psychosocial Functioning Questionnaire. 


\begin{tabular}{lllllll}
\hline Table 2 & \multicolumn{6}{l}{ Results of confirmatory factor analysis } \\
\hline$\chi^{2}$ & $\chi^{2} /$ df & GFI & NFI & RFI & CFI & RMSEA \\
\hline 403.121 & 3.125 & 0.888 & 0.924 & 0.910 & 0.947 & 0.085
\end{tabular}

CFI, Comparative Fit Index; df, degrees of freedom; GFI, Goodness of Fit Index; NFI, Non-normed Fit Index; RFI, Relative Fit Index; RMSEA, root mean square error of approximation.

the score of SDS had the highest correlation with the score of social functioning $(\mathrm{r}=0.695)$.

\section{DISCUSSION}

\section{Main findings}

Based on a theoretical construct of psychosocial functioning of depressed patients via literature analysis, open-ended patient questionnaires and interviews, clinical expert review, and three rounds of questionnaire administration and analysis, the PFQ scale for depressed patients was developed and has demonstrated good reliability and validity.

In this study, the psychosocial functioning of depressed patients was determined to be the ability of an individual to create relationships with others and society in a mutually pleasing manner, and the ability to achieve a healthy life independently. In the finalized questionnaire, the psychosocial functioning structure in depressed patients included three dimensions: psychological cognitive functioning, subjective well-being and social functioning. Of these, psychological cognitive functioning comprised the irrational beliefs and expectations of patients with depression; subjective well-being involved balancing positive and negative emotions and life satisfaction; and social functioning principally involved work performance, family relationships, interpersonal relationships, leisure activities, and social engagement.

For the finalized scale's reliability evaluation, this research examined the internal consistency reliability and the questionnaire's test-retest reliability. The outcome implied that the questionnaire had excellent internal consistency reliability $(\alpha=0.957)$ and test-retest reliability $(\mathrm{r}=0.840)$.

This research investigated the validity of the questionnaire via construct validity and criterion validity. Factor analysis and correlation analysis revealed that the PFQ had great construct validity. In the criterion validity test, one of the criteria was the recovery of a healthy life for patients with depression. Patients with less impaired psychosocial functioning can live a healthier life, as shown by the result $(r=-0.765)$. In addition, the PHQ-9, which is currently a more clinically accepted diagnostic and evaluation tool for depression, was applied as a criterion in this research. Studies have indicated that the PHQ-9 had a higher correlation with patients' functional impairment than other depression assessment scales (eg, Hamilton Rating Scale for Depression, Self-rating Depression Scale, and so on). ${ }^{16}$ This article suggested that high levels of psychosocial functioning were highly correlated with the severity of depression. In the meantime, Q-LESQ-SF, DAS and SDS were chosen to review the measurement of subjective well-being, psychological cognitive functioning, and social functioning, respectively. The outcome explained a meaningful correlation between these three questionnaires and PFQ. This demonstrated that all three dimensions of this questionnaire measured what they were intended to measure in advance .

In addition to good reliability and validity, the biggest advantage of the PFQ developed in this study is that it is specially designed for patients with MDD, and the questionnaire is concise and has fewer items. The connotation of psychosocial functioning varies among different groups where it is tested. Existing studies have found that the psychosocial functioning of patients with MDD includes psychological cognitive functioning, subjective well-being, interpersonal functioning, professional functioning, and so on. However, there is no single scale to evaluate the above functions. For example, SDS is the most widely used and concise scale for measuring functioning, but it can only evaluate the work, family and social functioning of patients, excluding psychological functioning such as psychological cognition functioning, and subjective well-being. Due to the lack of specific evaluation tools for patients with MDD, researchers usually choose multiple questionnaires to evaluate patients' psychosocial functioning, which leads to lengthy tests with numerous items.

\section{Research limitations}

This research also has some limitations that need to be addressed in subsequent studies. First, since the research adopted convenience sampling, the sample representativeness might be biased, so it is necessary to continue to expand the scope of subjects in the future. Also, a more

Table 3 Correlation coefficient between dimensions

Subjective well-being Psychological cognitive functioning Social functioning Total scores

\begin{tabular}{|c|c|c|c|c|}
\hline Subjective well-being & 1 & & & \\
\hline $\begin{array}{l}\text { Psychological cognitive } \\
\text { functioning }\end{array}$ & $0.807^{\star \star \star}$ & 1 & & \\
\hline Social functioning & $0.810^{\star \star \star}$ & $0.822^{\star \star \star}$ & 1 & \\
\hline Total scores & $0.936^{\star \star \star}$ & $0.914^{\star \star \star}$ & $0.957^{\star \star \star}$ & 1 \\
\hline
\end{tabular}

${ }^{* * *} \mathrm{p}<0.001$. 
Table 4 Correlation coefficients between PFQ and criterion questionnaires

\begin{tabular}{|c|c|c|c|c|}
\hline & Total PFQ & Subjective well-being & Psychological cognitive functioning & Social functioning \\
\hline Q-LES-Q-SF & $-0.870^{\star \star \star}$ & $-0.853^{\star \star \star}$ & $-0.787^{\star \star \star}$ & $-0.823^{\star \star \star}$ \\
\hline DAS & $0.663^{\star * *}$ & $0.599^{\star * *}$ & $0.662^{\star * *}$ & $0.623^{\star \star \star}$ \\
\hline
\end{tabular}

${ }^{* * \star} \mathrm{p}<0.001$.

DAS, Dysfunctional Attitudes Scale; PFQ, Psychosocial Functioning Questionnaire; Q-LES-Q-SF, Short Form of Quality of Life Enjoyment and Satisfaction Questionnaire; SDS, Sheehan Disability Scale.

standard control group is needed to further develop the norm of PFQ. Finally, although scholars agree that the psychosocial functioning of patients with MDD includes psychological functioning and social functioning, there is no consensus on the specific structure of psychosocial functioning. So the structure of this questionnaire requires further testing and improvement in research and practice.

\section{Research implications}

Patients with MDD have substantial impairment in psychosocial functioning, and impaired psychosocial functioning does not often completely recover even after the patient achieves a clinical remission of depressive symptoms. ${ }^{17}$ Improvement and recovery of psychosocial functioning are strongly associated with remission and relapse of MDD. ${ }^{16}$ This article describes a more relevant psychosocial functioning assessment tool for depressed patients, which helps to assess more comprehensively for treatment, recovery and relapse prevention of depression. Simultaneously, it increases attention to the recovery of patients' psychosocial functioning, thus advancing the quality of treatment, reducing the relapse rate, and decreasing the overall medical burden. Second, this questionnaire could implement an explicit and supportive method for assessing the efficacy of the psychotherapy for depression, thereby stimulating the development and use of psychological intervention programmes for depression.

Contributors XZ contributed to the development of $\mathrm{PFQ}$ and prepared the manuscript. HY contributed preparation of the manuscript. KD, CT and HW collected the data. NZ and HM contributed to the design of this study. NZ is the guarantor who accepts full responsibility for the work and/or the conduct of the study. All the authors have revised the manuscript for important intellectual content.

Funding This work was supported by the research project fund of the Health Care for Cadres in Jiangsu Province (BJ19005) and Key R\&D Plan of Jiangsu Province (Social Development, BE2015609).

Competing interests None declared.

Patient consent for publication Not required.

Ethics approval This study involves human participants and was approved by the Ethics Committee of Nanjing Brain Hospital Affiliated to Nanjing Medical University (reference no 2015-KY002). Participants gave informed consent to participate in the study before taking part.

Provenance and peer review Not commissioned; externally peer reviewed.
Data availability statement All data relevant to the study are included in the article or uploaded as supplemental information. Not applicable.

Open access This is an open access article distributed in accordance with the Creative Commons Attribution Non Commercial (CC BY-NC 4.0) license, which permits others to distribute, remix, adapt, build upon this work non-commercially, and license their derivative works on different terms, provided the original work is properly cited, appropriate credit is given, any changes made indicated, and the use is non-commercial. See: http://creativecommons.org/licenses/by-nc/4.0/.

\section{REFERENCES}

1 IsHak WW, James DM, Mirocha J, et al. Patient-reported functioning in major depressive disorder. Ther Adv Chronic Dis 2016;7:160-9.

2 Wang Z, Qu H, Zhong J, et al. Restoration of psychosocial functioning in remitted major depressive disorder patients: a 1-year longitudinal study. Compr Psychiatry 2020;102:152204.

$3 \mathrm{Ma} \mathrm{H}$, Yang H, Zhang N. Characteristics of psychosocial function recovery in convalescent depression and necessity of continuous psychotherapy. Chin J Behav Med Brain Sci 2011;20:1145-6.

4 Katz S, Ford AB, Moskowitz RW, et al. Studies of illness in the aged: the index of ADL: a standardized measure of biological and psychosocial function. JAMA 1963;185:914-9.

5 Yerxa EJ. 1966 Eleanor Clarke Slagle lecture. Authentic occupational therapy. Am J Occup Ther 1967;21:1-9.

6 Feragne MA, Longabaugh R, Stevenson JF. The psychosocial functioning inventory. Eval Health Prof 1983;6:25-48.

7 Bonder BR. Issues in assessment of psychosocial components of function. Am J Occup Ther 1993:47:211-6.

8 Whiffen VE, Gotlib IH. Comparison of postpartum and nonpostpartum depression: clinical presentation, psychiatric history, and psychosocial functioning. J Consult Clin Psychol 1993;61:485-94.

9 Clark LA, Vittengl JR, Kraft D, et al. Shared, not unique, components of personality and psychosocial functioning predict depression severity after acute-phase cognitive therapy. J Pers Disord 2003;17:406-30.

10 Lam RW, Filteau M-J, Milev R. Clinical effectiveness: the importance of psychosocial functioning outcomes. J Affect Disord 2011;132(Suppl 1):S9-13.

11 McQuaid JR, Marx BP, Rosen Ml, et al. Mental health assessment in rehabilitation research. J Rehabil Res Dev 2012;49:121-38.

12 Zheng T, Shi Y, Zhang N. Reliability and validity of Patient Health Questionnaire-9 in patients with stroke depression. Beijing Medicine 2013;35:352-6.

13 Lee Y-T, Liu S-I, Huang H-C, et al. Validity and reliability of the Chinese version of the Short Form of Quality of Life Enjoyment and Satisfaction Questionnaire (Q-LES-Q-SF). Qual Life Res 2014;23:907-16.

14 Leu S-H, Chou J-Y, Lee P-chin, et al. Validity and reliability of the Chinese version of the Sheehan Sisability Scale (SDS-C). Asia-Pacific Psychiatry 2015;7:215-22.

15 Chen Y, Xu J, Yan S. Preliminary study on reliability and validity of dysfunctional status assessment scale. Chin J Mental Health 1998:265-7.

16 Jha MK, Minhajuddin A, Greer TL, et al. Early improvement in psychosocial function predicts longer-term symptomatic remission in depressed patients. PLoS One 2016;11:e0167901.

17 Skärsäter I, Baigi A, Haglund L. Functional status and quality of life in patients with first-episode major depression. $J$ Psychiatr Ment Health Nurs 2006;13:205-13. 


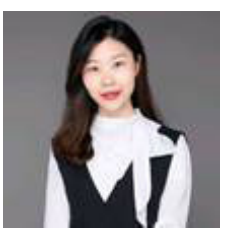

Xiujuan Zhang obtained a master's degree in psychology from Nanjing Normal University, China in 2016. As a postgraduate, she completed her master's thesis at the Nanjing Brain Hospital Affiliated to Nanjing Medical University in China. Her main research interest includes the recovery of the psychosocial functioning in patients with major depressive disorder. The development of a psychosocial functioning questionnaire for patients with major depressive disorder is an important part of her research. 